\title{
Gaussian beam evolution in nonlinear media of the Kerr type
}

\author{
P. Berczyński, ${ }^{* 1}$ Yu. A. Kravtsov, ${ }^{2}$ \\ ${ }^{1}$ Institute of Physics, West Pomeranian University of Technology, Szczecin 70-310, Poland, \\ ${ }^{2}$ Institute of Physics, Maritime University of Szczecin, Szczecin 70-500, Poland
}

Received March 07, 2013; accepted March 18, 2013, published March 31, 2013

\begin{abstract}
The paper generalizes paraxial complex geometrical optics (PCGO) for Gaussian beam (GB) propagation in nonlinear media of the Kerr type. PCGO deals with ordinary differential equations for the beam amplitude and for complex curvature of the wave front which describes the evolution of an axially symmetric GB in a Kerr type nonlinear medium. It is shown that PCGO readily provides the solutions of an NLS equation obtained earlier from the diffraction theory on the basis of an aberration-free approach. Besides reproducing the classica results of self-focusing, PCGO readily describes the influence of initial curvature of the wave front on the beam evolution in a medium of the Kerr type.
\end{abstract}

The great advantage of a complex geometrical optics method (CGO) is its ability to describe diffraction processes on the basis of ordinary differential equations. This property of CGO was demonstrated as far back as 40-50 years ago [1-3] (see also review papers [4-6] and Ch.5 in the book [7]).

In this paper we develop paraxial complex geometrical optics (PCGO) to describe the propagation and diffraction of axially symmetric Gaussian beams in nonlinear media of the Kerr type. This approach readily provides the basic results of nonlinear optics, obtained for Gaussian beams still 40-50 years ago [8, 9]. PCGO does not only reproduce former results, albeit in an easier way, but it also supplies a number of new results which are the subject of this paper. In particular, PCGO allows to include into analysis the initial curvature of the wave front and to study its influence on GB evolution in the media of the Kerr type.

For an axially symmetric wave beam in an axially symmetric nonlinear medium the PCGO method suggests the following solution

$$
u(\rho, z)=A \exp \left(i k_{0} \psi\right)=A(z) \exp \left[i k_{0}\left(z+B(z) \rho^{2} / 2\right)\right] .
$$

The real and imaginary parts of the complex curvature $B=B_{R}+i B_{I}$ determine the real curvature $\kappa$ of the wave front and the beam width $w$, correspondingly:

\footnotetext{
*E-mail: pawel.berczynski@ zut.edu.pl
}

$$
B_{R}=\kappa, \quad B_{I}=\frac{1}{k_{0} w^{2}},
$$

where $k_{0}=2 \pi / \lambda_{0}$ and $\lambda_{0}$ is the wavelength of the beam in vacuum. The PCGO method deals with the Riccati equation for complex curvature $\mathrm{B}$ :

$$
\frac{d B}{d z}+B^{2}=\alpha
$$

the equation for GB complex amplitude

$$
\frac{d A}{d z}+B A=0
$$

and the second order equation for GB width evolution,

$$
\frac{d^{2} w}{d z^{2}}-\alpha w=\frac{1}{k_{0}^{2} w^{3}}
$$

Parameter $\alpha$ for an axially symmetric medium equals:

$$
\alpha=\left.\frac{1}{2} \frac{\partial^{2} \varepsilon}{\partial \rho^{2}}\right|_{\rho=0} .
$$

The above equations were derived in paper [10]. In nonlinear media of the Kerr type the permittivity depends on the beam intensity $|u|^{2}$

$$
\varepsilon(\mathbf{r})=\varepsilon_{L I N}(\mathbf{r})+\varepsilon_{N L}|u(\mathbf{r})|^{2},
$$

where coefficient $\varepsilon_{N L}$ is assumed to be positive: $\varepsilon_{N L}>0$. Substituting wave field (1) into Eq. (7) one obtains

$$
\varepsilon(\rho, z)=\varepsilon_{L I N}(\rho, z)+\varepsilon_{N L}|A(z)|^{2} \exp \left(-\frac{\rho^{2}}{w(z)^{2}}\right)
$$


In fact, the PCGO method treats a nonlinear medium of the Kerr type as a smoothly inhomogeneous medium whose profile is additionally modulated by GB parameters $w$ and $A$. Therefore, the PCGO method presented in the past for a linear case can be also applicable for nonlinear media of the Kerr type. In accordance with the relation

$$
w^{2}|A|^{2}=w^{2}(0)|A(0)|^{2},
$$

one can present Eq. (8) as:

$$
\varepsilon(\rho, z)=\varepsilon_{L I N}(\rho, z)+\frac{\varepsilon_{N L}|A(0)|^{2} w^{2}(0)}{w^{2}(z)} \exp \left(-\frac{\rho^{2}}{w^{2}(z)}\right) .
$$

Taking into account the relation in Eq. (6), the alpha parameter in Eqs. $(3,5)$ can be given as:

$$
\alpha=\alpha_{L I N}+\alpha_{N L}
$$

where the first term refers to the linear medium,

$$
\alpha_{L I N}=\left.\frac{1}{2} \frac{d^{2} \varepsilon_{L I N}}{d \rho^{2}}\right|_{\rho=0}
$$

and the second term accounts for nonlinear processes:

$$
\alpha_{N L}=\left.\frac{1}{2} \frac{\varepsilon_{N L}|A(0)|^{2} w^{2}(0)}{w^{2}(z)} \frac{d^{2}}{d \rho^{2}} \exp \left(-\frac{\rho^{2}}{w^{2}(z)}\right)\right|_{\rho=0}=-\frac{\varepsilon_{N L}|A(0)|^{2} w^{2}(0)}{w^{4}(z)}
$$

In conditions when the contribution of a linear term in Eq. (11) is negligibly small, the Riccati equation (3) takes the form

$$
\frac{d B}{d z}+B^{2}=\alpha_{N L}=-\frac{\varepsilon_{N L}|A(0)|^{2} w^{2}(0)}{w^{4}(z)}
$$

This equation describes the GB evolution under the influence of nonlinear processes without any contribution of linear refraction. Equation (5) for the beam width evolution in a medium of the Kerr type takes the form

$$
\frac{d^{2} w}{d z^{2}}+\frac{\varepsilon_{N L}|A(0)|^{2} w^{2}(0)}{w^{3}}=\frac{1}{k_{0}^{2} w^{3}} .
$$

Introducing a dimensionless beam width $f=w / w(0)$, Eq. (15) can be rewritten as

$$
\frac{d^{2} f}{d z^{2}}=-\frac{1}{f^{3}}\left(\frac{1}{L_{N L}^{2}}-\frac{1}{L_{D}^{2}}\right)
$$

where $L_{D}=k_{0} w^{2}(0)$ is the diffraction length and $L_{N L}=w(0) / \sqrt{\varepsilon_{N L}|A(0)|^{2}}$ is the characteristic nonlinear scale. Equation (16) can be also presented as

$$
\frac{d^{2} f}{d \zeta^{2}}=-\frac{1}{f^{3}}\left(\frac{L_{D}^{2}}{L_{N L}^{2}}-1\right)
$$

where dimensionless distance $\zeta=z / L_{D}$ is involved. It can be proved that

$$
\frac{L_{D}^{2}}{L_{N L}^{2}}=\frac{P}{P_{c r i t}}
$$

where $P=\frac{1}{8} c w^{2}(0)|A(0)|^{2}$ is the total beam power and $P_{c r i t}=\frac{1}{8} \frac{c}{k_{0}^{2} \varepsilon_{N L}}$ is the critical power. As a result, the equation for GB width evolution in a nonlinear medium of the Kerr type takes the form:

$$
\frac{d^{2} f}{d \zeta^{2}}+\frac{1}{f^{3}}\left(\frac{P}{P_{\text {crit }}}-1\right)=0
$$

Integrating once Eq. (19) and assuming that $\left.\frac{d f}{d z}\right|_{z=0}=0$, which corresponds to the GB with a plane initial wave front, one obtains the following solution

$$
f^{2}=1-\frac{z^{2}}{L_{D}^{2}}\left(\frac{P}{P_{c r i t}}-1\right)
$$

The above PCGO result is in total agreement with the solution of the nonlinear parabolic equation presented in the pioneering papers [8,9]. Thus, the PCGO method reproduces the classical results of nonlinear optics but in a simpler and more illustrative way.

The first integral of Eq. (16) takes the form

$$
\frac{1}{2}\left(\frac{d f}{d z}\right)^{2}+\frac{1}{2}\left(\frac{1}{L_{D}^{2}}-\frac{1}{L_{N L}^{2}}\right) \frac{1}{f^{2}}=C
$$

In accordance with [10], the value $d f / d z$ at $z=0$ presents the squared initial wave front curvature:

$$
\left.\left(\frac{d f}{d z}\right)^{2}\right|_{z=0}=\left.\frac{1}{w^{2}(0)}\left(\frac{d w}{d z}\right)^{2}\right|_{z=0}=\kappa^{2}(0)
$$


In accordance with Eq. (18), Eq. (21) takes the following form

$$
f^{2}\left(\frac{d f}{d \zeta}\right)^{2}+\left(\frac{P}{P_{c r i t}}-1-\kappa^{2}(0) L_{D}^{2}\right) f^{2}=\frac{P}{P_{c r i t}}-1
$$

Taking advantage of differential relation $\left[\left(f^{2}\right)^{\prime}\right]^{2}=4 f^{2} f^{\prime 2}$ in the above equation, one obtains:

$$
\frac{1}{4}\left(\frac{d f^{2}}{d \zeta}\right)^{2}+\left(\frac{P}{P_{c r i t}}-1-\kappa^{2}(0) L_{D}^{2}\right) f^{2}=\frac{P}{P_{c r i t}}-1
$$

and differentiating once (24) we have :

$$
\frac{1}{2}\left(\frac{d f^{2}}{d \zeta}\right)\left(\frac{d^{2} f^{2}}{d \zeta^{2}}\right)+\left(\frac{P}{P_{c r i t}}-1-\kappa^{2}(0) L_{D}^{2}\right) \frac{d f^{2}}{d \zeta}=0
$$

As a result, the equation for beam width evolution in a nonlinear medium of the Kerr type takes the form

$$
\frac{d^{2} f^{2}}{d \zeta^{2}}=2\left(1+\kappa^{2}(0) L_{D}^{2}-\frac{P}{P_{c r i t}}\right)
$$

Then Eq. (26), has the following solution

$$
f^{2}=\frac{z^{2}}{L_{D}^{2}}\left(\kappa^{2}(0) L_{D}^{2}-\frac{P}{P_{c r i t}}+1\right)+2 \kappa(0) z+1 .
$$

It is worth analyzing the following partial cases:

1) Sub-critical regime: $P<P_{c r i t}$. In the case of a divergent beam, answering to positive curvature $\kappa(0)>0$, diffraction widening prevails over self-focusing effect and GB width increases. For a convergent beam, corresponding to $\kappa(0)<0$, the beam width initially decreases reaching its minimum value

$$
w_{\min }=w(0) \sqrt{\frac{1-P / P_{c r i t}}{\kappa^{2}(0) L_{D}^{2}-P / P_{c r i t}+1}}
$$

and next the diffraction widening dominates and the beam width starts increasing.

2) Critical regime $P=P_{c r i t}\left(L_{D}=L_{N L}\right)$. In this case diffraction divergence and self-focusing effects compensate each other and $f^{2}$ depends only on the sign of $\kappa(0)$ :

$$
f^{2}=\kappa^{2}(0) z^{2}+2 \kappa(0) z+1=[(\kappa(0) z+1)]^{2}
$$

As a result, the beam width $w$ changes as a linear function of distance $z$ :

$$
w=w(0)[\kappa(0) z+1]
$$

In this solution, factor $\kappa(0)>0$ leads to a growth of the GB width, whereas for $\kappa(0)=0$, one obtains a stationary solution with $\mathrm{w}=\mathrm{w}(0)$. For $\kappa(0)<0$ the beam is focused.

3) Over-critical regime $P>P_{c r i t}$. Let us involve the characteristic beam power $P^{\prime}=P_{c r i t}\left[1+\kappa^{2}(0) L_{D}^{2}\right\rfloor$. When the total power $P$ is equal to $P^{\prime}$, quadratic term in solution (27) happens to be zero and parameter $f^{2}$ becomes a linear function of $z$. As a result beam width is equal to

$$
w=w(0) \sqrt{2 \kappa(0) z+1} .
$$

For the GB power $P$, which is greater than the critical power and smaller than characteristic power $P_{c r i t}<P<P_{c r i t}\left[1+\kappa^{2}(0) L_{D}^{2}\right]$, a positive value of the initial wave front curvature $\kappa(0)=1 / L_{D}$ eliminates the collapse and, as a result, the beam width increases. One can notice that a negative value of initial phase front curvature $\kappa(0)=-1 / L_{D}$ only enhances the beam collapse effect.

\section{References}

[1] Yu.A. Kravtsov, Radiophys. Quant. Electronics 10, 719 (1967)

[2] J.B. Keller, W. Streifer, J. Opt. Soc. Am. 61, 40 (1971).

[3] G.A. Deschamps, Electron. Lett. 7(23), 684 (1971).

[4] Yu.A. Kravtsov, G.W. Forbes, A.A. Asatryan, Progress in Optics 39, 3 (1999).

[5] S.J. Chapman, J.M. Lawry, J.R. Ockendon, R.H. Tew, SIAM Rev. 41, 417 (1999).

[6] Yu.A. Kravtsov, P. Berczynski, Stud. Geophys. Geod. 51(1), 1 (2007).

[7] Yu.A. Kravtsov, Geometrical Optics in Engineering Physics (Alpha Science International, UK, 2005).

[8] S.A. Akhmanov, A.P. Sukhorukov, R V. Khokhlov, Soviet Phys. Uspekhi 10, 609 (1968).

[9] S.A. Akhmanov, R.V. Khokhlov, A.P. Sukhorukov, Self-focusing, Selfdefocusing and Self-modulation of Laser Beams in Laser Handbook (F.T. Arecchi and E.O. Shulz-Dubois, Eds; Elsevier, New York, 1972).

[10] P. Berczyński, Yu. Kravtsov, Phot. Lett. Poland 4(1), 26 (2012). 